\title{
High Performance Low Power (HPLP) Driver Fatigue Detection System
}

\author{
Vijayalaxmi \\ Departement of Electronics and Communication Engineering, VITS, Deshmukhi \\ D.Elizabath Rani \\ Departement of Electroincs and Instrumentation Engineering, GITAM University, Vishakapatnam
}

\begin{abstract}
A High Performance Low Power (HPLP) Fatigue Detection system is developed using Non-Intrusive vision based approach. The system uses Universal Serial Bus (USB) Logitech Pro 9000 camera which is mounted inside vehicle to monitor driver's face continuously. In Vision based HPLP Fatigue Detection system Hardware plays a very important role in terms of cost, size, power consumption and speed. In this research, Texas Instrument (TI) based DM3730 Processor -1GHz ARM Cortex-A8 core board is used which has four USB ports, RS-232 port, Camera port and Expansion port to interface 7" LCD capacitive Touch screen. The board uses up to $2 \mathrm{~W}$ of power and can be powered using USB connector or $5 \mathrm{~V}$ of power supply. Due to low power consumption, no separate Heat Sinks are required. ARM Cortex-A8 runs on Linux, Minix, Android, WinCE, Symbian operating system. In this work, Linux version Ubuntu 11.10 is ported on to microSD card of $8 \mathrm{~GB}$. The camera is connected to one of the USB port of the board which captures frames @30fps.
\end{abstract}

Keywords: DM3730,Fatigue,ARM,Ubuntu,Non-intrusive.

\section{INTRODUCTION}

Achieving high performance in image processing is a challenging task in real time. Most of the image processing algorithms are compact and repeated over large number of data and need lot of computation. Development in digital imaging technology made the computation load on image processing grow in terms of image size and algorithm complexity. This situation, has led to select high end processor for performing complex image processing task. Developing image processing based system needs to combat and meet requirements such as size, cost, power consumption and time-to-market by selecting a proper processor.

Performing image processing task on computer is slow because it executes repetitive operations by fetchexecute-cycle. DSPs or ASSPs can be used in consumer image processing products. But to perform complex image processing task, DSPs need more parallel unit. Hence such powerful DSPs become expensive. Dedicated hardware and parallel processing increases the performance of image processing system with low cost. Digital Signal Processor (DSP) is a specialized image processor used to perform image processing task. To increase the speed and efficiency of image processing algorithms, image processor often use parallel computing.

The digital image/vision processing systems are the combination of image processing software and computer hardware. Achieving high performance is a challenging task in image processing applications. Many image processing applications are described compactly with few functions but they are repeated over large amount of time. Hence demand more computational efforts. Image processing is performed on mainframe computer system, workstations, or Personal Computers (PCs) [1]. The main difference is in speed at which the computer processes millions of instructions per second (MIPS).

A mainframe computer system uses $>32$-bit Central Processing Unit (CPU), the advantage of this system is its high speed performance and its disadvantage is its coarse output. Workstations uses $>32$-bit CPU and are relatively inexpensive and consists of Reduced Instruction Set Computer (RISC), own operating system, CPU and digital image processing software. A PC system uses 16 to 32-bit CPU with digital image processing software. These machines cost $<\$ 1,500$ including a colour monitor with an 8-bit look-up table.

The load on the image processing algorithms is increasing due to fast growth in imaging technology. This scenario leads to choose high-end processor for performing digital image processing task. The commonly used processors for performing image processing task are Microcontroller, FPGA, ARM processor.

A small computer integrated on single Silicon chip is termed as microcontroller which has inbuilt memory (RAM, ROM), processor core, and programmable input/output peripherals. Microcontrollers are preferred over Microprocessor while designing embedded applications. The clock frequency at which Microcontrollers operate is at $4 \mathrm{KHz}$ with less power consumption. Microcontroller with higher clock speeds and more power consumption are used to serve critical roles and behave like Digital Signal Processor (DSP).

A DSP is a special processor with optimized architecture to increase the operational speed of digital signals. Better processor with more speed and memory is required to perform image processing applications. To handle, 
image processing complexity, DSP's with more number of parallel units are required. The cost of such powerful DSP's is not economical and their performance gets deviated from the actual requirements. The powerful processor may not serve the basic requirements of image processing applications such as cost, power consumption, size, time to market.

Texas Instruments' (TI), combine DSP and ARM, creating best platform for developing applications with improved speed and efficiency. Combining ARM processing with DSP processing improves flexibility, real time performance and reduced cost and power. Power control systems, machine vision and tracking applications, require high end processors; hence DSP+ARM processors are useful. This processor is useful for running signal and image processing algorithms on DSP apart from ARM processor. DSP and ARM processor on a single chip provides on-chip network connectivity (EMCA and USB), memory interfaces and standard interfaces which improves system performance and reduce system cost [8].

DM3730 processor is fabricated on single chip Texas Instrument's advanced 45-nm technology with improved performance. ARM architecture provides best Graphics with less power consumption. DM3730 $1 \mathrm{GHz}$ processor comes in $0.4 \mathrm{~mm}$ pitch POP (Package on Package) with memory mounted on top of the processor.

\section{FLOWCHART OF HPLP FATIGUE DETECTION SYSTEM}

High Performance Low Power (HPLP) fatigue detection system flowchart is shown in Figure 1. HPLP Fatigue detection system processes real time video stream to identify drivers' different fatigue level by detecting the frames in which eyes are open/closed.

As shown in Figure 1, as soon as the board power is ON, firstly it will check for all the connected peripherals working condition such as keyboard, mouse, camera, GSM module and speakers. If anyone of this is not working properly, then the board is reset using RESET button for rebooting. After successful verification of all peripherals, Camera starts capturing video stream. For the video stream, the system checks for lighting. For poor lighting condition, a set of 50 LED's can be used to illuminate driver's face.

This set of 50 LED's as source of illumination for testing purpose is used in the Laboratory and is fixed such that it does not illuminate the drivers face directly. If the light source directly falls on drivers face than it would be difficult for the driver to drive the vehicle due to reflection. Hence, it is used only for testing purpose.

The captured video stream is converted into frames, to perform image processing task to estimate driver's fatigue based on Eye blink rate and Yawning. The proposed image processing algorithm for Fatigue estimation is discussed in next section. The driver fatigue is estimated using Eye blink rate, the warning is issued to the driver, if still driver fails to stop the vehicle or take necessary step, than an SMS alert will be sent to the registered number.

\section{REAL TIME HPLP FATIGUE DETECTION SYSTEM}

Real time HPLP detection system is developed using image processing where driver's face is recorded through camera continuously. Most of the image processing based system depend on intensity changes, hence it is required that background should not contain any object with strong intensity changes. The camera connected may capture highly reflective object behind the driver's seat as an eyes. The design of the prototype is developed assuming that inside the vehicle there is no direct light and background is uniform.

As per the requirement, when the ambient light is poor (night time), a light source must be present to compensate the effect of poor light. Since it is a prototype, 50 LED's light source is used which illuminates the driver's face during poor lighting condition. This light source is fixed such that it will not illuminate driver's eyes directly. If the source of light is not taken into consideration, the camera with lights can be used for capturing the driver's image which will illuminate the image.

The HPLP Fatigue detection system consists of Logitech Pro 9000 USB camera to capture driver's face. The camera is placed in front of driver's face, approximately $25 \mathrm{~cm}$ away from the face. Position of the camera is such that, it must meet the following criteria

i. Majority of the captured frame should contain driver's face

ii. Drivers face should be at the centre of the frame. 


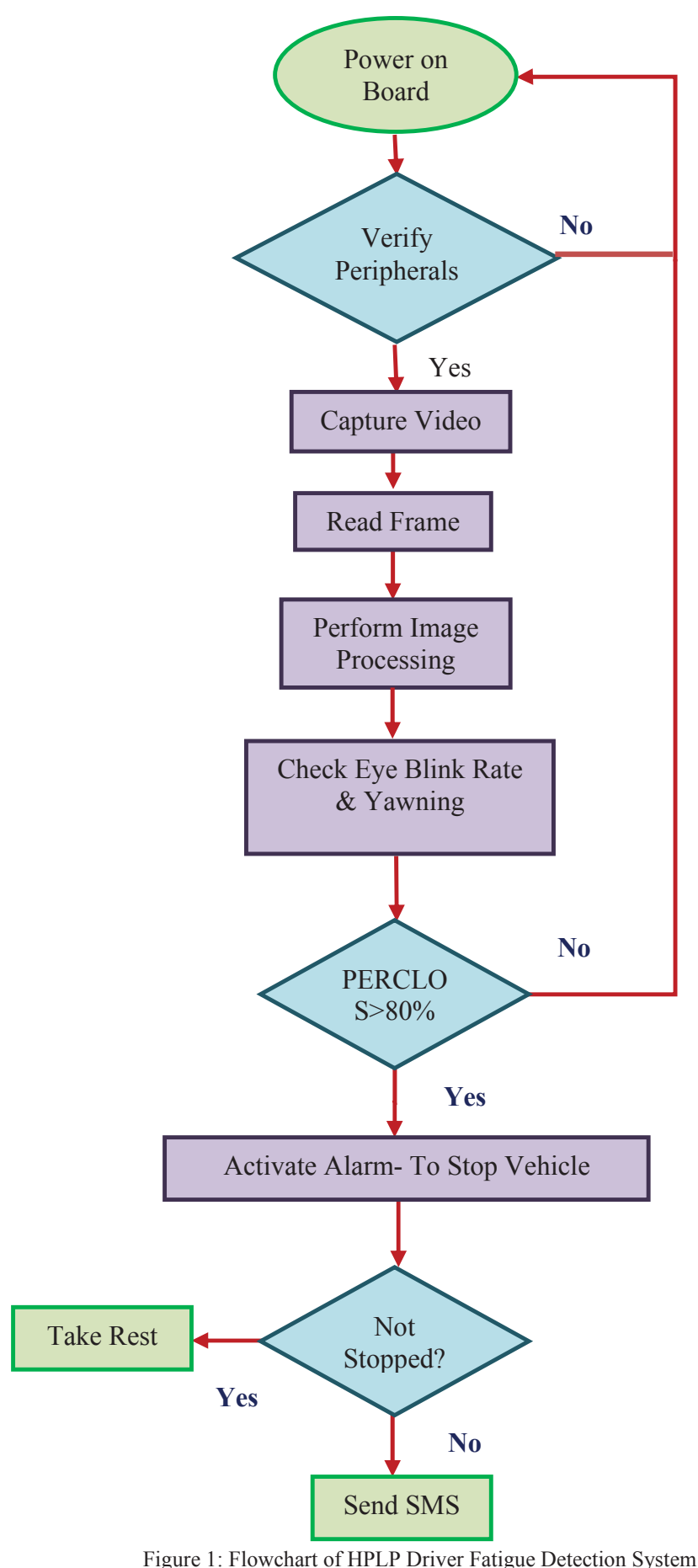

The above discussed two criteria reduce the complexity of identifying face and eye certain extent. Figure 2 shows the prototype of the HPLP fatigue detection system. In real time the entire setup is tested by placing HPLP Fatigue detection system in a vehicle as shown in Figure 3. 


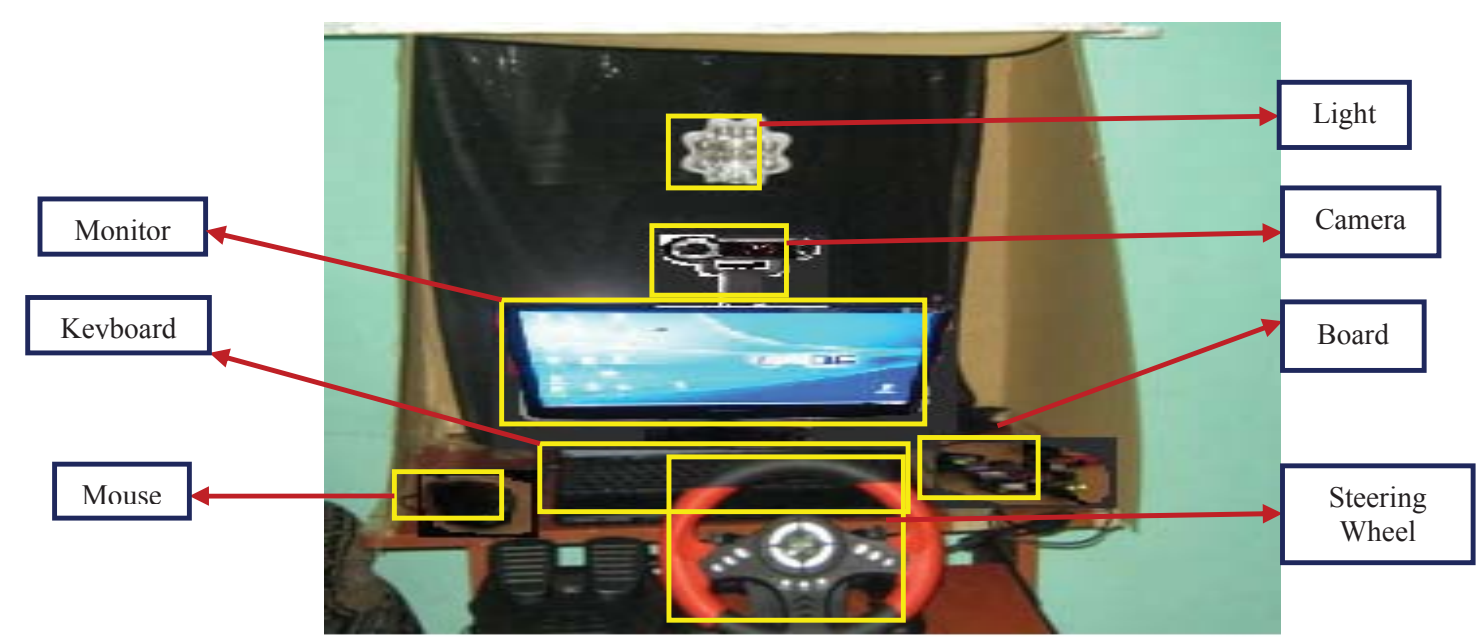

Figure 2: Prototype of HPLP Fatigue Detection System

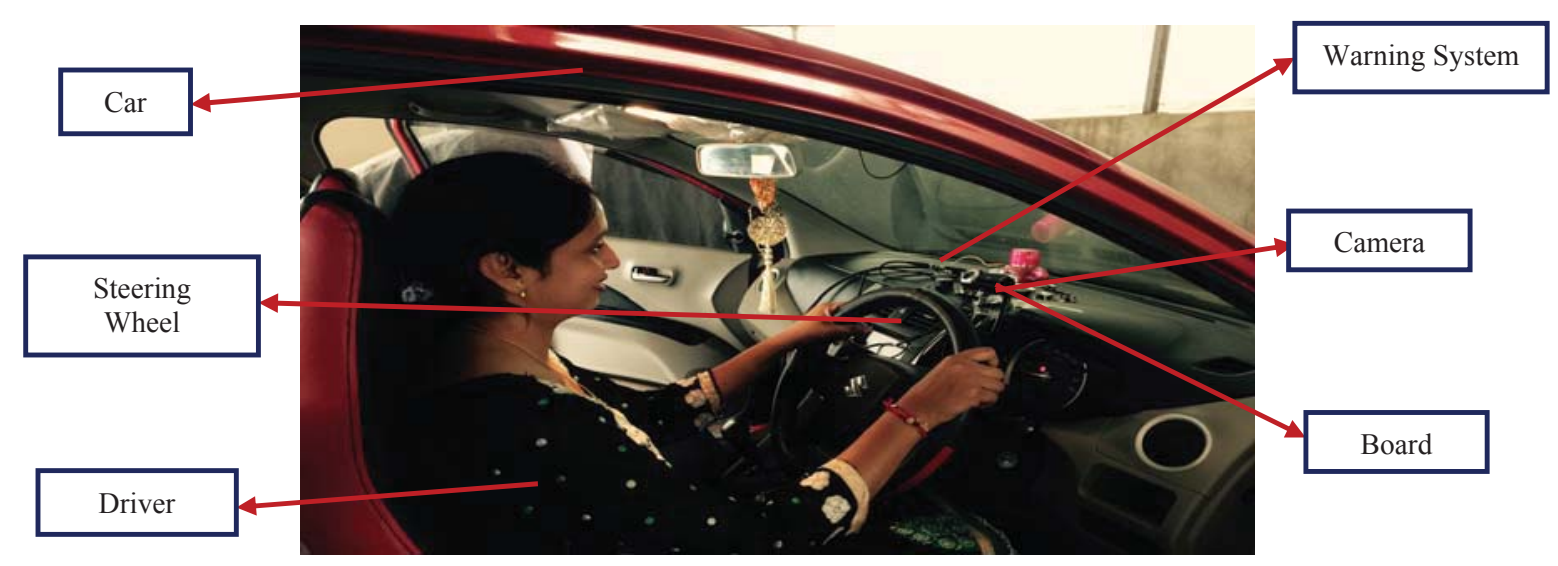

Figure 3: Real Time Implementation of HPLP Fatigue Detection System

In real time set-up, the Logitech Pro 9000 camera is fixed to dashboard near to steering wheel at $25 \mathrm{~cm}$ away from driver. The board and remaining peripherals are placed onto the dashboard as shown

Figure 3 without disturbing or distracting the driver.

\section{COMPARISON OF HPLP DRIVER FATIGUE DETECTION SYSTEM WITH EXISTING TECHNOLOGIES}

This section compares and contrast, Proposed HPLP Driver Fatigue Detection system with existing systems in terms of traction and engineering criteria by user, ' $V$ 'represents condition of system satisfy objective, a ' $\mathrm{X}$ ' represents that the objective is not satisfied, a ' $\bullet$ ' denotes that more data is needed. The comparison of HPLP Driver Fatigue Detection system with other existing technologies based on few criteria is shown in Table 4.9. 
Table 1: Comparison of Drive Fatigue Detection System

\begin{tabular}{|c|c|c|c|c|c|c|}
\hline Objective & $\begin{array}{c}\text { Attention } \\
\text { Technology } \\
\text { Driver } \\
\text { Fatigue } \\
\text { Monitor }\end{array}$ & $\begin{array}{l}\text { Delphi } \\
\text { Driver } \\
\text { State } \\
\text { Monitor }\end{array}$ & $\begin{array}{c}\text { Seeing } \\
\text { Machines } \\
\text { faceLAB }^{\mathrm{TM}}\end{array}$ & $\begin{array}{c}\text { SmartEye } \\
\text { Machines } \\
\text { faceLAB }^{\mathrm{TM}}\end{array}$ & $\begin{array}{c}\text { Smart Eye } \\
\text { Pro 3.0 }\end{array}$ & $\begin{array}{c}\text { HPLP Driver } \\
\text { Fatigue Detection } \\
\text { System-Proposed } \\
\text { Method }\end{array}$ \\
\hline $\begin{array}{l}\text { System must operate } \\
\text { with accuracy, } \\
\text { reliably in all } \\
\text { environments }\end{array}$ & $\mathbf{X}$ & • & • & • & • & $\sqrt{ }$ \\
\hline $\begin{array}{l}\text { System must operate } \\
\text { under all weather } \\
\text { conditions }\end{array}$ & $\sqrt{ }$ & $\sqrt{ }$ & $\sqrt{ }$ & $\sqrt{ }$ & $\sqrt{ }$ & $\sqrt{ }$ \\
\hline $\begin{array}{l}\text { System should } \\
\text { minimize false alarms }\end{array}$ & $\bullet$ & • & • & $\bullet$ & $\bullet$ & $\sqrt{ }$ \\
\hline $\begin{array}{l}\text { System must be robust } \\
\text { and economical }\end{array}$ & $\bullet$ & $\bullet$ & $\bullet$ & $\bullet$ & $\bullet$ & $\sqrt{ }$ \\
\hline $\begin{array}{l}\text { System must be non- } \\
\text { intrusive }\end{array}$ & $\sqrt{ }$ & $\sqrt{ }$ & $\sqrt{ }$ & $\sqrt{ }$ & $\sqrt{ }$ & $\sqrt{ }$ \\
\hline $\begin{array}{l}\text { System must } \\
\text { accommodate } \\
\text { spectacles }\end{array}$ & $\mathbf{X}$ & $\sqrt{ }$ & $\sqrt{ }$ & $\sqrt{ }$ & $\sqrt{ }$ & $\sqrt{ }$ \\
\hline $\begin{array}{l}\text { Proper Warning } \\
\text { System }\end{array}$ & $\sqrt{ }$ & $\sqrt{ }$ & $\sqrt{ }$ & $\sqrt{ }$ & $\sqrt{ }$ & $\sqrt{ }$ \\
\hline $\begin{array}{l}\text { System should work in } \\
\text { real time }\end{array}$ & $\sqrt{ }$ & $\sqrt{ }$ & $\sqrt{ }$ & $\sqrt{ }$ & $\sqrt{ }$ & $\sqrt{ }$ \\
\hline $\begin{array}{l}\text { System should provide } \\
\text { feedback }\end{array}$ & $\sqrt{ }$ & $\sqrt{ }$ & • & • & • & $\sqrt{ }$ \\
\hline $\begin{array}{l}\text { System should take } \\
\text { minimum training } \\
\text { data }\end{array}$ & $\sqrt{ }$ & $\sqrt{ }$ & $\sqrt{ }$ & $\sqrt{ }$ & $\sqrt{ }$ & • \\
\hline $\begin{array}{l}\text { System must not } \\
\text { distract/disturb the } \\
\text { driver }\end{array}$ & $\sqrt{ }$ & $\sqrt{ }$ & $\sqrt{ }$ & $\sqrt{ }$ & $\sqrt{ }$ & $\sqrt{ }$ \\
\hline
\end{tabular}




\section{CONCLUSION}

Driver Fatigue Detection system is one of the machine vision application which deals with continuous video stream and converting to frames per second. The Processor with $1.5 \mathrm{GHz}$ floating and fixed point DSP is capable of analysing these images in real-time with high performance. This high-performance processor comes with set of peripherals such as PCI express, Ethernet, two 32-bit DDR3 memory interface operating at $1600 \mathrm{MHz}$ makes this DFD System a High Performance. Hence, all the above features make this DFD System High Performance and Low Power system.

\section{REFERENCES}

[1] Russ, J.C., "The Image Processing Handbook”, Boca Raton, FL:CRC Press,445p,(1992).

[2] Bruner,J.S. and Tagiuri,R., "The perception of people", Handbook of Social Psycology, 2(17), (1954).

[3] Saito.S, "Does fatigue exist in a quantitative measurement of eye movements?", Ergonomics,35(5),pp.607-615., (1992).

[4] Artaud et.al, Mabbott et.al, Lavergne et.al, Vitabile et.al, Eskandarian.A \& R.Sayed in , "Monitoring the response of drivers",( 1994),(1999),(1996),2007-08),(2005).

[5] Otmani,S., Pebayle,T., Roge,J., Muzet,A., "Effect of driving duration and partial sleep deprivation on subsequent alertness and performance of car drivers," Physiological Behaviour, (2005).

[6] Martin Gallagher, "Development of a driver alert system for road safety", in (2006-07).

[7] Chin T.L., Che J.C., Bor S.L., Shao H.H., Chih F.C., Wang I.J., "A real-time wireless brain-computer interface system for drowsiness detection”, IEEE Transactions Biomedical Circuits Systems, 4,pp.214-222,(2010).

[8] Daniel Allred, Gustavo Martinez," Maximizing the Power of ARM ® with DSP", White paper Texas Instruments, (October 2010).

[9] Jackson, P., Hilditch, C., Holmes, A., Reed, N., Merat, N. \& Smith, L. "Fatigue and Road Safety: A Critical Analysis of Recent Evidence", Road Safety Web Department for Transport, Publication No. 21, (2011). 